\section{Endoleak after an Open Abdominal Aortic Aneurysm Repair}

\section{Abstract}

Although endoleaks have been well described after endovascular abdominal aortic aneurysm (AAA) repair, their occurrence after open repair is much less common. This case report describes a patient who had a leak at the proximal anastomotic site of the graft after open AAA repair. The patient was a 78 year old male patient who presented to our facility with bright red blood from the rectum at approximately 3 months after an open AAA repair at an outside facility. On computed tomographic angiography, a leak was detected at the proximal anastomotic site and resembled a type I endoleak that may occur after endovascular repair. The patient was treated through endovascular technique with the placement of two proximal extension cuffs. The endoleak was not observed on follow-up imaging, and the patient has been asymptomatic since treatment. Endoleaks after open AAA repair are rare, but they can be treated through an endovascular approach, with proximal extension cuff placement to seal the leak.

Keywords: Anastomotic leak; Endovascular repair; Open abdominal aortic aneurysm repair; Proximal extension cuff; Type 1 endoleak

Abbreviations: AAA: Abdominal Aortic Aneurysm; CTA: Computed Tomographic Angiography; EVAR: Endovascular Aortic Repair; PAA: Para Anastomotic Aneurysm
Charles G Stoneburner', Gregory Frey ${ }^{1}$ and Albert G Hakaim²

1 Department of Radiology, Mayo Clinic, Jacksonville, Florida, USA

2 Department of Surgery, Mayo Clinic, Jacksonville, Florida, USA

Corresponding author:

Albert G Hakaim

” hakaim.albert@mayo.edu

Department of Surgery, Mayo Clinic, 4500

San Pablo Rd, Jacksonville, FL 32224,

Florida, United States.

Tel: $+904-953-2000$

Citation: Stoneburner CG, Frey G, Hakaim AG. Endoleak after an Open Abdominal Aortic Aneurysm Repair. Journal of Vascular \& Endo Surgery. 2016, 1:1.

\section{Introduction}

Endoleaks occurring after endovascular aortic repair (EVAR) have been well described. However, patients who have undergone open abdominal aortic aneurysm (AAA) repair also may have associated complications observable on imaging. These complications include aortoenteric and arterioenteric fistulas, graft limb thrombosis, prosthetic graft infection, anastomotic compromise leading to leak, and para anastomotic aneurysms (PAAs) [1, 2]. Although PAAs have been well described and may be associated with a leak at the proximal or distal portion of the graft, they are typically seen long after the original repair, usually about 10 years postoperatively [3]. This case report describes a patient who presented from an outside facility and was found to have an endoleak after undergoing open AAA repair approximately 3 months prior. Although PAAs have been associated with endoleaks, an endoleak occurring so close to the postoperative period is less common to observe in a patient who has had open AAA repair.

\section{Case Report}

The patient was a 78 year old man with past medical history of AAA status post open repair in August 2014 at an outside facility, long-term lupus anticoagulation treatment with warfarin, pulmonary embolus, and peripheral vascular disease. $\mathrm{He}$ presented to our facility in November 2014 with severe weakness and stools with bright red blood. He first began to have symptoms 5 days before his presentation at our clinic, when he had severe back pain as he drove from New York to Florida. However, the patient reported that the pain had resolved by the next day, and he did not seek medical care at that point. He presented to our clinic approximately 1 week after these initial symptoms, when he began to have bright red blood from the rectum and had 2 bloody stools. During his interview, the patient noted that his surgery involved complications but was unable to provide any details.

The patient received computed tomographic angiography (CTA) shortly after his presentation; the CTA was notable for status 
post open AAA repair with a now-enlarged aneurysm measuring $7.0 \times 6.8 \mathrm{~cm}$. In addition, an accumulation of contrast agent was apparent within the aortic sac on arterial phase imaging, suggestive of an active endoleak (Figures 1 and 2). The patient had been seen at our facility previously and had a prior computed tomography scan that showed the AAA measuring $5.2 \mathrm{~cm} \times 4.4$ $\mathrm{cm}$ in February 2013 before his repair. There was concern for contrast medium extending from the proximal end of the sac distally, which was suspicious for the pattern of leakage seen in a type I endoleak after EVAR.

The next day, the patient underwent aortography, and no type II endoleak was found. The patient then underwent placement of 2 proximal extension cuffs, measuring $32 \mathrm{~mm} \times 39 \mathrm{~mm}$ and $36 \mathrm{~mm} \times 50 \mathrm{~mm}$, to treat the endoleak. CTA at 2 days after this intervention found that the aneurysm had decreased in size to $5.3 \mathrm{~mm} \times 6.5 \mathrm{~cm}$ with no evidence of a new endoleak (Figure 3). On follow - up 1 month later, the aneurysm had further decreased to $5.8 \mathrm{~cm} \times 6.0 \mathrm{~cm}$ with no evidence of recurrence (Figure 4).

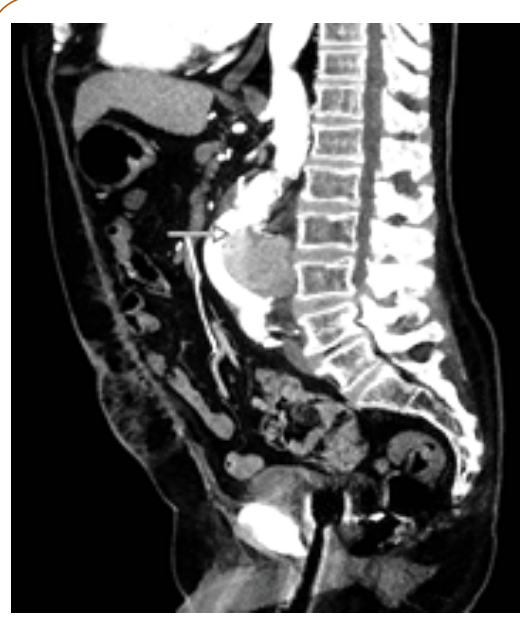

Figure 1 Sagittal image of extravasated contrast agent from the proximal portion of the graft. This finding (arrow) is indicative of an active endoleak.

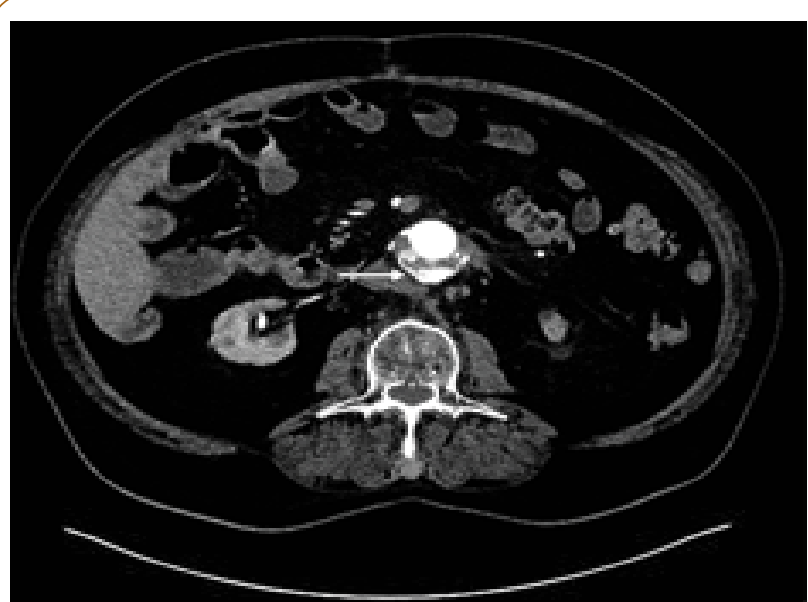

Figure 2 Axial image of extravasated contrast agent from the proximal portion of the graft. Arrow points to area of interest.

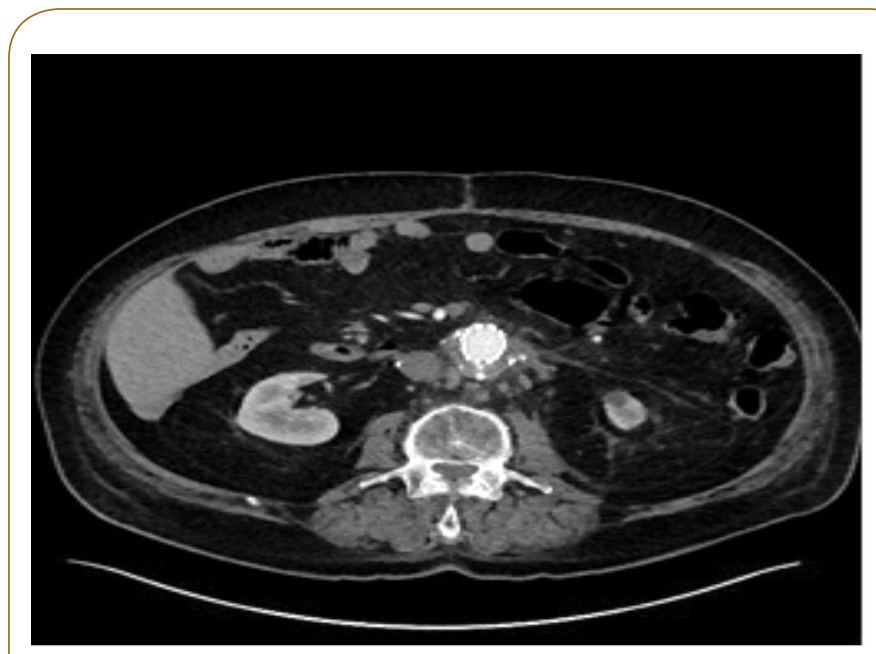

Figure 3 Axial image of the patient shortly after the insertion of 2 proximal extension cuffs.

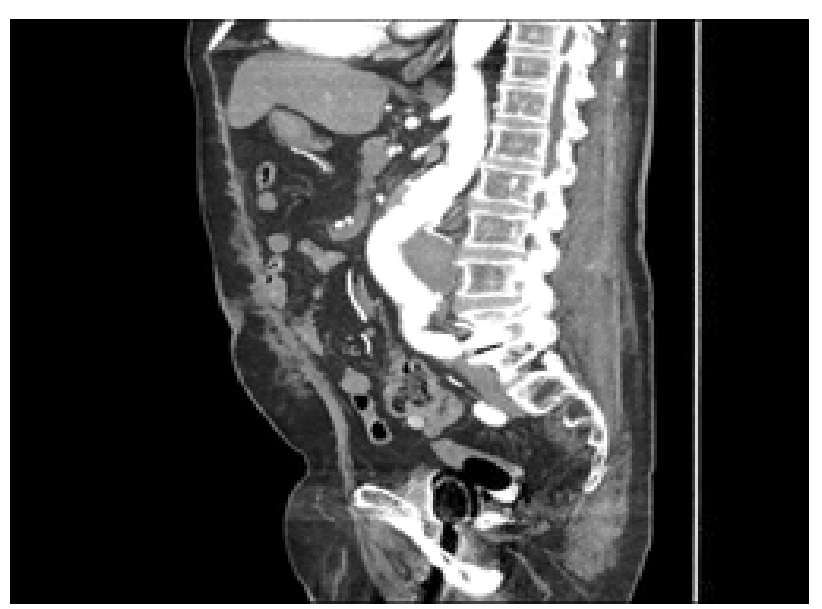

Figure 4 Sagittal image from an examination performed 1 month after the intervention to correct the condition shown in Figure 1. The endoleak is no longer present and the aneurysm size has decreased.

The patient reported that he had been doing well and had no recurrence of symptoms.

\section{Discussion}

Endoleaks after EVAR have been well described. However, the example in the present report shows a rare case of a type I endoleak after an open repair. Such endoleaks have been described, but they are less frequently reported. The relatively short period from operation to presentation of this endoleak also makes it a rare event. The patient presented approximately 3 months after the open repair operation. This complication has been cited as occurring in less than $1 \%$ of patients. However, this percentage may be greater because it pertains to symptomatic patients, and many endoleaks that occur after open repair may resolve spontaneously, similar to their reported course in endovascular stenting [4]. Patients who present with symptoms that include abdominal or back pain in the postoperative period 
should be considered at risk for an endoleak [2]. Studies have shown that PAAs with leakage may be asymptomatic [1], so it seems plausible that acute endoleaks may be asymptomatic as well.

Digital subtraction angiography is regarded as the best method for detecting an endoleak because of its high spatial and temporal resolution that allows the demonstration of flow direction. However, CTA and color flow Doppler imaging also can be used [2]. Doppler may be helpful to further examine endoleak classification when using CTA. When contrast medium is seen outside the graft and within the aneurysmal sac on CTA, this finding is the most reliable indication of an endoleak [2].

Although the exact mechanism of endoleak after open repair is not entirely understood, it is believed to be due to either loosening of the sutures, suture breakage, or disruption of the suture union due to poor vessel wall quality, which can be observed when suturing along calcified arteries [4]. The exact mechanism of leak in the present case was not fully understood. Imaging before the procedure does show that the abdominal aorta was calcified, although the level of calcification does not appear to be markedly increased compared with that of most candidates for AAA repair. No direct radiologic evidence showed that the sutures became loose or were broken.

The data most relevant for repair of an endoleak after an open EVAR are those from the repair of open para-anastomatic aneurysms. For open repair of PAAs, the mortality rate varies substantially depending on whether a patient is symptomatic or asymptomatic; it ranges from up to $70 \%$ in symptomatic patients to $14 \%$ in asymptomatic patients [5]. These relatively high mortality rates are due to the increased difficulty of operating in previously explored surgical fields, as well as the increased age of the patients compared with their age at the original operation. However, investigators have demonstrated that endovascular repair of PAAs decreases mortality rates compared with open repair when the patient's anatomy is suitable [3].

Nolz et al. [6] observed mortality rates of $0 \%$ for asymptomatic patients and $20 \%$ for symptomatic patients in endovascular repair of PAAs. Morrissey et al. [7] recorded early mortality rates after endovascular repair at 3.6\%. In addition, the endovascular approach allows the surgeon to gain access at a location far from the operative site, with no need to clamp the abdominal aorta or dissect scarring from the previous operations in the abdomen and pelvis [3].

As patients age, they are more likely to have had multiple abdominal operations. These prior surgeries can increase the difficulty of the operation and, potentially, the risk of damage to abdominal structures, thereby placing this patient group at increased risk. Given the small amount of information on the treatment of endoleak occurring recent to the operation, this information on treatment of PAAs was applied for the patient in this case report, which was treated with endovascular insertion of 2 proximal extension cuffs and has done well at follow-up of more than 6 months.

Endoleaks are traditionally associated with EVAR and have 5 different classifications. The present case most resembles a type I endoleak; these endoleaks occur when a leak at one of the graft attachment sites allows for arterial flow to enter the space outside the graft. Given that arterial flow is pressurized, this alternative flow pattern creates the opportunity for aneurysm expansion and potentially places the patient at risk for aneurysm rupture [8]. Type I and type III endoleaks warrant prompt intervention after they are detected; however, many type II endoleaks may be at risk for increasing intrasac pressure as well. Type I endoleaks may be seen in the immediate postoperative period or may have delayed presentation because of changes in graft orientation due to decreased size of the aneurysm [9].

The best way to mitigate the risk of a type I endoleak is to ensure that the patient's anatomy is within the accepted limits and the appropriate graft is used. Care must be taken to ensure that the vessels have the appropriate size, shape, and angulation to safely permit endovascular repair [8]. In addition, the infrarenal neck must be long enough and the distal insertion sites adequate for stent placement.

Rosen and Green [8] advised that a proximal attachment site of less than $1 \mathrm{~cm}$ with angulation greater than $60^{\circ}$ is highly likely to encounter complications. Typically, the first attempt at repair is angioplasty in the area of the leak, in an attempt to seal off the arterial flow. If this technique is not successful, a balloon expandable bare metal stent is typically used in the area. The increased force of the balloon - expandable stent gives the best chance of sealing off the leak [10]. Finally, if this second technique also is not effective, a stent graft may be placed over the area as long as it does not interfere with the adherent portion of the original stent [10].

\section{Conclusion}

The present case report shows a rare example of an endoleak occurring after open EVAR. Although the exact cause of the endoleak was never determined, 2 proximal cuffs placed with endovascular technique sealed the leak. The patient tolerated the procedure well and has been doing well at follow - up visits. 


\section{References}

1 Duvnjak S, Andersen PE, Larsen KE, Roeder O (2014) Endovascular repair of postoperative vascular graft related complications after aorto-iliac surgery. Int Angiol 33: 386-391.

2 Nayeemuddin M, Pherwani AD, Asquith JR (2012) Imaging and management of complications of open surgical repair of abdominal aortic aneurysms. Clin Radiol 67: 802-814.

3 Gawenda M, Zaehringer M, Brunkwall J (2003) Open versus endovascular repair of para-anastomotic aneurysms in patients who were morphological candidates for endovascular treatment. J Endovasc Ther 10: 745-751.

4 Chan CL, Ray SA, Taylor PR, Fraser SC, Giddings AE (2000) Endoleaks following conventional open abdominal aortic aneurysm repair. Eur J Vasc Endovasc Surg 19: 313-317.

5 Locati P, Socrate AM, Costantini E (2000) Paraanastomotic aneurysms of the abdominal aorta: a 15-year experience review. Cardiovasc Surg 8: 274-279.
6 Nolz R, Gschwendtner M, Julg G, Plank C, Beitzke D, et al. (2012) Anastomotic pseudoaneurysms after surgical reconstruction: outcomes after endovascular repair of symptomatic versus asymptomatic patients. Eur J Radiol 81: 1589-1594.

7 Morrissey NJ, Yano OJ, Soundararajan K, Eisen L, McArthur C, et al. (2001) Endovascular repair of para-anastomotic aneurysms of the aorta and iliac arteries: preferred treatment for a complex problem. J Vasc Surg 34: 503-512.

8 Rosen RJ, Green RM (2008) Endoleak management following endovascular aneurysm repair. J Vasc Interv Radiol 19: S37-S43.

9 Bashir MR, Ferral H, Jacobs C, McCarthy W, Goldin M (2009) Endoleaks after endovascular abdominal aortic aneurysm repair: management strategies according to CT findings. AJR Am J Roentgenol 192: W178-W186.

10 White SB, Stavropoulos SW (2009) Management of Endoleaks following Endovascular Aneurysm Repair. Semin Intervent Radiol 26: 33-38. 